\title{
THREAD ALGEBRA AND RISK ASSESSMENT SERVICES
}

\author{
JAN A. BERGSTRA, INGE BETHKE, AND ALBAN PONSE
}

\begin{abstract}
Threads as contained in a thread algebra emerge from the behavioral abstraction from programs in an appropriate program algebra. Threads may make use of services such as stacks, and a thread using a single stack is called a pushdown thread. Equivalence of pushdown threads is decidable. Using this decidability result, an alternative to Cohen's impossibility result on virus detection is discussed and some results on risk assessment services are proved.
\end{abstract}

$\S 1$. Introduction. This paper is about thread algebra $[2,6]$. Threads are processes tailored to describe sequential program behaviour and emerge from the behavioral abstraction of sequential programs. A basic thread models a finite program behaviour to be controlled by some execution environment: upon each action (e.g. a request for some service), a reply true or false from the environment determines further execution. Any execution trace of a basic thread ends either in the (successful) termination state or in the deadlock state. Both these states are modeled as special thread constants. Regular threads extend basic threads by comprising loop behaviour, and are reminiscent of flowcharts [14, 12]. Threads may make use of services, i.e., devices that control (part of) their execution by consuming actions, providing the appropriate reply, and suppressing observable activity. Regular threads using the service of a single stack are called pushdown threads. Apart from the distinction between deadlock and termination, pushdown threads are comparable to pushdown automata or pushdown processes as described by Stirling [17] or Burkart and Steffen [10].

First, we recall from our companion paper [3] that equivalence of pushdown threads is decidable, and we provide a sketch of our proof. Then we elaborate on Cohen's impossibility result on virus detection [11] (in that 1984 paper, the term computer virus was coined). Whereas Cohen showed that a test predicate that decides whether a program executes (and spreads) a virus cannot exist, we proposed in [9] a more modest test that can be used to forecast whether the execution of a thread has no security hazard. This is decidable for regular threads (as argued in [9]), and also for shrat-safe pushdown threads (as argued in this paper). In our approach, a security

Meeting

Edited by Unknown

(c) 1000 , Association For Symbolic Logic 
hazard is modeled as the occurrence of a certain action in a thread. We define a service SHRAT (security hazard risk assessment tool) that provides the replies to such tests. The idea is as follows: a security hazard is modeled by an action risk and the security hazard risk test as sh.ok. In case SHRAT replies true to

$$
\text { if sh.ok then } P \text { else } Q \text {, }
$$

$P$ will not execute risk and execution continues with $P$. In the other case (reply false), $Q$ will be executed instead because $P$ would execute risk (there is no security hazard risk assessment of $Q$ ). A major point is whether $P$ itself may or may not execute sh.ok tests. If $P$ is regular, this is not a problem and we prove that SHRAT is correct. In the case that $P$ is a pushdown thread, correctness only follows if $P$ is shrat-safe, i.e., contains no occurrences of both sh.ok and risk (this is a decidable property).

Our approach offers an alternative to that of Cohen in his well-known paper [11] which shows the impossibility of a test action that reacts on two arguments $P$ and $Q$ at the same time. More precisely, Cohen considers a decision procedure $\mathrm{D}$ (a predicate on program texts) that determines whether a program executes (and spreads) a virus. Then Cohen's impossibility result is established by the program $\mathrm{C}$ defined by

$$
\mathrm{C}=\text { if } \neg \mathrm{D}(\mathrm{C}) \text { then } P \text { else } Q \text {, }
$$

where $P$ executes a virus, and $Q$ is virus-free.

§2. Threads and services. In this section we recall the definitions of basic threads and regular threads. Furthermore we discuss services that may be used by a thread, and we consider the use-operator, which defines how a thread uses a service.

2.1. Threads. Basic thread algebra $[6]^{1}$, BTA, is tailored for the description of sequential program behaviour. Based on a finite set of actions $A$, it has the following constants and operators:

- the termination constant S,

- the deadlock or inaction constant D,

- for each $a \in A$, a binary postconditional composition operator $\_\unlhd a \unrhd$. We use action prefixing $a \circ P$ as an abbreviation for $P \unlhd a \unrhd P$ and take $\circ$ to bind strongest.

The operational intuition behind thread algebra is that each action represents a command which is to be processed by the execution environment of a thread. More specifically, an action is taken as a command for a service offered by the environment. The processing of a command may involve a

\footnotetext{
${ }^{1}$ In [5], basic thread algebra is introduced under the name basic polarized process algebra.
} 
change of state of this environment. At completion of the processing of the command, the service concerned produces a reply value true or false to the thread under execution. The thread $P \unlhd a \unrhd Q$ will then proceed as $P$ if the processing of $a$ yielded the reply true indicating successful processing, and it will proceed as $Q$ if the processing of $a$ yielded the reply false.

BTA can be equipped with a partial order and an approximation operator in the following way:

1. $\sqsubseteq$ is the partial ordering on BTA generated by the clauses

(a) for all $P \in \mathrm{BTA}, \mathrm{D} \sqsubseteq P$, and

(b) for all $P_{1}, P_{2}, Q_{1}, Q_{2} \in \mathrm{BTA}, a \in A$,

$$
P_{1} \sqsubseteq Q_{1} \& P_{2} \sqsubseteq Q_{2} \Rightarrow P_{1} \unlhd a \unrhd P_{2} \sqsubseteq Q_{1} \unlhd a \unrhd Q_{2} .
$$

2. $\pi: \mathbb{N} \times$ BTA $\rightarrow$ BTA is the approximation operator determined by the equations

(a) for all $P \in \mathrm{BTA}, \pi(0, P)=\mathrm{D}$,

(b) for all $n \in \mathbb{N}, \pi(n+1, \mathrm{~S})=\mathrm{S}, \pi(n+1, \mathrm{D})=\mathrm{D}$, and

(c) for all $P, Q \in \mathrm{BTA}, n \in \mathbb{N}$,

$$
\pi(n+1, P \unlhd a \unrhd Q)=\pi(n, P) \unlhd a \unrhd \pi(n, Q) .
$$

We further write $\pi_{n}(P)$ instead of $\pi(n, P)$.

The operator $\pi$ finitely approximates every thread in BTA. That is, for all $P \in \mathrm{BTA}$,

$$
\exists n \in \mathbb{N} \pi_{0}(P) \sqsubseteq \pi_{1}(P) \sqsubseteq \cdots \sqsubseteq \pi_{n}(P)=\pi_{n+1}(P)=\cdots=P .
$$

Every thread in BTA is finite in the sense that there is a finite upper bound to the number of consecutive actions it can perform. Following the metric theory of [1] in the form developed as the basis of the introduction of processes in [4], BTA has a completion $\mathrm{BTA}^{\infty}$ which comprises also the infinite threads. Standard properties of the completion technique yield that we may take $\mathrm{BTA}^{\infty}$ as the cpo consisting of all so-called projective sequences. That is,

$$
\mathrm{BTA}^{\infty}=\left\{\left(P_{n}\right)_{n \in \mathbb{N}} \mid \forall n \in \mathbb{N}\left(P_{n} \in \mathrm{BTA} \& \pi_{n}\left(P_{n+1}\right)=P_{n}\right)\right\}
$$

with

$$
\left(P_{n}\right)_{n \in \mathbb{N}} \sqsubseteq\left(Q_{n}\right)_{n \in \mathbb{N}} \Leftrightarrow \forall n \in \mathbb{N} P_{n} \sqsubseteq Q_{n}
$$

and

$$
\left(P_{n}\right)_{n \in \mathbb{N}}=\left(Q_{n}\right)_{n \in \mathbb{N}} \Leftrightarrow \forall n \in \mathbb{N} P_{n}=Q_{n} .
$$

For a detailed account of this construction see [2]. In this cpo structure, finite linear recursive specifications represent continuous operators having as unique fixed points regular threads, i.e., threads which can only reach 
finitely many states. A finite linear recursive specification over BTA is a set of equations

$$
X_{i}=t_{i}(\bar{X})
$$

for $i \in I$ with $I$ some finite index set and all $t_{i}(\bar{X})$ of the form $\mathrm{S}$, D, or $X_{i_{l}} \unlhd a_{i} \unrhd X_{i_{r}}$ for $i_{l}, i_{r} \in I$.

EXAMPLE 2.1.1. We define the regular threads

1. $a \circ b \circ \mathrm{D}$,

2. $a \circ b \circ \mathrm{S}$ and

3. $(a \circ b)^{\infty}$ (this informal notation is explained below)

as the fixed points for $X_{1}$ in the specifications

1. $X_{1}=a \circ X_{2}, X_{2}=b \circ X_{3}, X_{3}=\mathrm{D}$,

2. $X_{1}=a \circ X_{2}, X_{2}=b \circ X_{3}, X_{3}=\mathrm{S}$,

3. $X_{1}=a \circ X_{2}, X_{2}=b \circ X_{1}$, respectively.

Both $a \circ b \circ \mathrm{D}$ and $a \circ b \circ \mathrm{S}$ are finite threads; $(a \circ b)^{\infty}$ is the infinite thread corresponding to the projective sequence $\left(P_{n}\right)_{n \in \mathbb{N}}$ with $P_{0}=\mathrm{D}, P_{1}=a \circ \mathrm{D}$ and $P_{n+2}=a \circ\left(b \circ P_{n}\right)$. Observe that $a \circ b \circ \mathrm{D} \sqsubseteq a \circ b \circ \mathrm{S}, a \circ b \circ \mathrm{D} \sqsubseteq(a \circ b)^{\infty}$, but $a \circ b \circ \mathrm{S} \nsubseteq(a \circ b)^{\infty}$.

Convention 2.1.2. In reasoning with finite linear recursive specifications, we shall from now on identify variables and their fixed points. For example, we say that $P$ is the regular thread defined by $P=a \circ P$ instead of stating that $P$ equals the fixed point for $X$ in $X=a \circ X$.

2.2. Services. A service is a component of an execution architecture for threads that can be used to determine the reply to an action. In [7] various services (called state machines in that paper) were considered, as well as their possible role in thread execution. A service is a pair $\langle\Sigma, F\rangle$ consisting of a set $\Sigma$ of so-called co-actions and a reply function $F$. The reply function $F$ of a service $\langle\Sigma, F\rangle$ is a mapping that gives for each sequence of co-actions in $\Sigma^{+}$the reply produced by the service. This reply is a boolean value true or false.

EXAMPle 2.2.1 (Stack). One of the services that will occur in what follows is the stack $S=\langle\Sigma, F\rangle$ with $\Sigma=\{$ push: $i$, topeq: $i$, empty, pop $\mid i \in$ $I\}$ for some finite set $I$, where push: $i$ pushes $i$ onto the stack and yields reply true, the action topeq: $i$ tests whether $i$ is on top of the stack, empty tests whether the stack is empty, and pop pops the stack if it is non-empty with reply true and yields the reply false otherwise (leaving the stack empty). By $S(\alpha)$ we denote a stack with contents $\alpha \in I^{*}$ with the leftmost element of $\alpha$ on top in case $\alpha \neq \epsilon$ with $\epsilon$ the empty stack contents. In Example 3.1.1 we return to the use of a stack as a service. 
In order to provide a specific description of the interaction between a thread and a service, we will use for actions the general notation c.a where $\mathrm{c}$ is the so-called channel or focus and a is a co-action. For example, we write s.pop to denote the action which pops a stack via channel s.

For a service $\mathcal{S}=\langle\Sigma, F\rangle$ and a finite thread $P$, we define $P$ using the service $\mathcal{S}$ via channel $c$, notation $P / c \mathcal{S}$, by the following rules:

$$
\begin{aligned}
\mathrm{S} / \mathrm{c} \mathcal{S} & =\mathrm{S}, \\
\mathrm{D} / \mathrm{c} \mathcal{S} & =\mathrm{D}, \\
\left(P \unlhd \mathrm{c}^{\prime} . \mathrm{a} \unrhd Q\right) / \mathrm{c} \mathcal{S} & =(P / \mathrm{c} \mathcal{S}) \mathrm{c}^{\prime} \cdot \mathrm{a} \unrhd(Q / \mathrm{c} \mathcal{S}) \text { if } \mathrm{c}^{\prime} \neq \mathrm{c}, \\
(P \unlhd \mathrm{c} . \mathrm{a} \unrhd Q) / \mathrm{c} \mathcal{S} & =P / \mathrm{c} \mathcal{S}^{\prime} \text { if } \mathrm{a} \in \Sigma \text { and } F(\mathrm{a})=\text { true } \\
(P \unlhd \mathrm{c} . \mathrm{a} \unrhd Q) / \mathrm{c} \mathcal{S} & =Q / \mathrm{c} \mathcal{S}^{\prime} \text { if } \mathrm{a} \in \Sigma \text { and } F(\mathrm{a})=\text { false } \\
(P \unlhd \mathrm{c} . \mathrm{a} \unrhd Q) / \mathrm{c} \mathcal{S} & =\mathrm{D} \text { if } \mathrm{a} \notin \Sigma,
\end{aligned}
$$

where $\mathcal{S}^{\prime}=\left\langle\Sigma, F^{\prime}\right\rangle$ with $F^{\prime}(\sigma)=F($ a $\sigma)$ for all co-action sequences $\sigma \in \Sigma^{+}$. Note that actions that use a service $\mathcal{S}$ are not observable. The use operator is expanded to infinite threads $P$ by stipulating

$$
P / c \mathcal{S}=\left(\pi_{n}(P) / \mathrm{c} \mathcal{S}\right)_{n \in \mathbb{N}} .
$$

As a consequence, $P / c \mathcal{S}=\mathrm{D}$ if for every $n, \pi_{n}(P) / \mathrm{c} \mathcal{S}=\mathrm{D}$.

EXAMPLE 2.2.2. We consider again the threads $a \circ b \circ \mathrm{D}, a \circ b \circ \mathrm{S}$ and

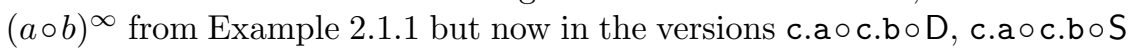
and (c.a $\circ$ c.b $)^{\infty}$ for some channel c and service $\mathcal{S}=\langle\{\mathrm{a}, \mathrm{b}\}, F\rangle$. Then $(\mathrm{c} . \mathrm{a} \circ \mathrm{c} . \mathrm{b} \circ \mathrm{D}) / \mathrm{c} \mathcal{S}=\mathrm{D}$ and $(\mathrm{c} . \mathrm{a} \circ \mathrm{c} . \mathrm{b} \circ \mathrm{S}) / \mathrm{c} \mathcal{S}=\mathrm{S}$, but $(\mathrm{c} . \mathrm{a} \circ \mathrm{c} . \mathrm{b})^{\infty} / \mathrm{c} \mathcal{S}=\mathrm{D}$.

§3. Pushdown threads and decidable equivalence. In this section we consider pushdown threads, i.e., regular threads that use a stack. Then, we recall from our paper [3] that equivalence of pushdown threads is decidable and sketch a proof of this fact.

3.1. Pushdown threads. In the next example we show that the use of services may turn regular threads into non-regular ones.

EXAMPLE 3.1.1. Let $\{a, b$, s.push:1, s.pop $\} \subseteq A$, where the last two actions refer to the stack $S$ defined in Example 2.2.1 with $I=\{1\}$. By the defining equations for the use operator it follows that for any thread $P$ and $\sigma \in\{1\}^{*}$,

$$
(\text { s.push: } 1 \circ P) / \mathrm{s} S(\sigma)=P / \mathrm{s} S(1 \sigma) .
$$

Furthermore, it easily follows that

$$
(P \unlhd \mathrm{s} . \mathrm{pop} \unrhd \mathrm{S}) / \mathrm{s} S(\sigma)= \begin{cases}\mathrm{S} & \text { if } \sigma=\epsilon \text { (the empty sequence) } \\ P / \mathrm{s} S(\rho) & \text { if } \sigma=1 \rho .\end{cases}
$$


Now consider the regular thread $Q$ defined by ${ }^{2}$

$$
\begin{aligned}
& Q=(\text { s.push: } 1 \circ Q) \unlhd a \unrhd R, \\
& R=b \circ R \unlhd \text { s.pop } \unrhd \mathrm{S} .
\end{aligned}
$$

Then for all $\sigma \in\{1\}^{*}$,

$$
\begin{aligned}
Q / \mathrm{s} S(\sigma) & =((\text { s.push: } 1 \circ Q) \unlhd a \unrhd R) / \mathrm{s} S(\sigma) \\
& =(Q / \mathrm{s} S(1 \sigma)) \unlhd a \unrhd(R / \mathrm{s} S(\sigma)), \\
R / \mathrm{s} S(1 \sigma) & =b \circ R / \mathrm{s} S(\sigma), \\
R / \mathrm{s} S(\epsilon) & =\mathrm{S} .
\end{aligned}
$$

It is not hard to see that $Q / \mathrm{s} S(\epsilon)$ is an infinite thread with the property that for all $n \in \mathbb{N}$, a trace of $n+1 a$-actions produced by $n$ positive and one negative reply on $a$ is followed by $n b$-actions and $\mathrm{S}$. This yields an nonregular thread: if $Q / \mathrm{s} S(\epsilon)$ were regular, it would be a fixed point of some finite linear recursive specification, say with $k$ equations. But specifying a trace containing $k b$-actions followed by $\mathrm{S}$ already requires $k+1$ linear equations $X_{1}=b \circ X_{2}, \ldots, X_{k}=b \circ X_{k+1}, X_{k+1}=\mathrm{S}$, which contradicts the assumption. So $Q / \mathrm{s} S(\epsilon)$ is not regular.

We call a regular thread that uses a stack as described in Example 2.2.1 a pushdown thread. In what follows we assume that pushdown threads are given with help of a distinguished identifier from a finite linear recursive specification $\mathcal{F}$ and a stack over some fixed alphabet. The equations in $\mathcal{F}$ may contain actions that address the stack via the use-application $/ \mathbf{s}$.

3.2. Decidable equivalence. From our companion paper [3] we quote the following result:

Theorem 3.2.1. Equivalence of pushdown threads is decidable.

This theorem follows from a reduction to the dpda-equivalence problem whose decidability was proved by Sénizergues $[15,16]$. Here we provide only a sketch, a detailed proof can be found in [3].

The idea is to use a transformation from pushdown threads to dpda's such that the identity

$$
P / \mathbf{s} S(\alpha)=Q / \mathrm{s} S(\beta)
$$

holds if and only if the identity

$$
L\left(\mathcal{A}, P^{\prime} \alpha^{\prime}\right)=L\left(\mathcal{A}, Q^{\prime} \beta^{\prime}\right)
$$

holds, where the latter identity expresses that for the derived dpda $\mathcal{A}$, the language accepted by 'configuration' $P^{\prime} \alpha$ equals the one accepted by

\footnotetext{
${ }^{2}$ Note that a linear recursive specification of $Q$ requires (at least) five equations.
} 
configuration $Q^{\prime} \beta^{\prime}$. The transformation described in [3] consists of five steps and uses the dpda-equivalence result as formulated by Stirling [18] because this is closer to our setting:

1. Transform $P / \mathrm{s} S(\alpha)$ and $Q / \mathrm{s} S(\beta)$ such that initially the stacks are non-empty (also if one of $\alpha$ and $\beta$ is the empty string), and such that upon their termination the stack is empty. The reason for this step stems from the fact that language acceptance for dpda's is defined on configurations of the form $R \alpha$ where $R$ is a 'state' and $\alpha$ is a nonempty stack contents. A word $w$ is in the accepted language iff the dpda in initial state $R$ empties the stack by performing the transitions whose labels form $w$.

2. Replace occurrences of D by loops that fill the stack (e.g., replace $P_{i}=\mathrm{D}$ by $P_{i}=\mathrm{s} \cdot \mathrm{push}: j \circ P_{i}$ for some $j \in I$ ). The reason for this step is that $\mathrm{D}$ has no equivalent in the dpda-equivalence result.

3. Normalize infinite traces: replace each equation $P_{i}=P_{l} \unlhd a \unrhd P_{r}$ by $P_{i}=\overline{\mathrm{S}} \unlhd b \unrhd\left(P_{l} \unlhd a \unrhd P_{r}\right)$ with $b$ an action that occurs not in $P$ and $Q$. Here $\bar{S}$ is the thread that first empties the stack and then terminates $(\bar{S}$ is also used in step 1$)$. The reason for this step is that each infinite trace becomes interlarded with exits $b$, and is thus characterized by finite traces which in turn are subject to dpda language acceptance.

4. Construction of an associated pushdown automaton (pda). The specifications of the so far transformed $P(\alpha)$ and $Q(\beta)$ admit a straightforward definition of a pda whose transitions are deterministic. The only remaining problem is that the $\epsilon$-transitions (that stem from stack actions) need not pop the stack, as required by the decidability result in $[18]$.

5. Construction of a dpda in which the $\epsilon$-transitions only pop the stack. The pda thus obtained is transformed by changing its transition rules for $\epsilon$. Those that do not pop the stack are either swallowed by an observable transition and yield a new transition rule, or form a loop, in which case they can be omitted. This step preserves language acceptance and concludes the transformation.

We will exploit this decidability result by replacing certain equations in the definition of the regular thread that underlies a pushdown thread, i.e. in the definition of $P$ when considering $P / \mathrm{s} S(\alpha)$. For example, it is decidable whether a pushdown thread is normed, i.e., has the option to terminate (to end in S): let a linear recursive specification

$$
\mathcal{F}=\left\{P_{i}=t_{i}(\vec{P}) \mid i=1, \ldots, n\right\}
$$

be given (and thus a repertoire of stack actions and external actions). Replace each equation $P_{i}=\mathrm{S} \in \mathcal{F}$ by $\bar{P}_{i}=a \circ \bar{P}_{i}$ and overline all remaining identifiers. Then $P_{k} / \mathrm{s} S(\alpha)$ is normed $\Leftrightarrow P_{k} / \mathbf{s} S(\alpha) \neq \bar{P}_{k} / \mathbf{s} S(\alpha)$. 
REMARK 3.2.2. Interestingly, inclusion of pushdown threads is not decidable (although two pushdown threads are equivalent if they are included in each other). This follows from a reduction to the halting problem for Minsky machines - an approach also taken in Jančar et al. [13]. A detailed proof is recorded in [3].

§4. Security hazard risk assessment. In this section we consider the possibility that a pushdown thread uses a service that supports forecasting of certain future behaviour. In [8] various such services are studied (e.g., the halting problem and "rational agents") and in [9] we discuss a rather specific case: a service SHRAT (security hazard risk assessment tool). In this paper we provide a detailed construction of SHRAT for regular threads and a proof of its correctness. Finally, we consider SHRAT for pushdown processes and distinguish the case of shrat-safe threads.

4.1. A definition of SHRAT. We model a security hazard in a pushdown thread $\mathcal{P}$ as the execution of an action risk. Furthermore, $\mathcal{P}$ may contain a test action sh.ok that can use the service SHRAT to forecast whether risk will be executed: SHRAT replies true to

$$
\mathcal{Q} \unlhd \text { sh.ok } \unrhd \mathcal{R}
$$

if $\mathcal{Q}$ does not execute risk, and false if $\mathcal{Q}$ does execute the action risk (and then $\mathcal{R}$ is executed instead). In order to model forecasting, we first define the residual thread of a pushdown thread $\mathcal{P}$ as the thread that remains after zero or more actions of $\mathcal{P}$ have been executed:

Definition 4.1.1. Let $\mathcal{P}$ be a pushdown thread. We write $\mathcal{Q} \in \operatorname{Res}(\mathcal{P})$ whenever $\mathcal{Q}$ is a residual thread of $\mathcal{P}$ :

- $\mathcal{P} \in \operatorname{Res}(\mathcal{P})$,

- $\mathcal{P} \in \operatorname{Res}(\mathcal{P} \unlhd a \unrhd \mathcal{Q})$,

- $\mathcal{Q} \in \operatorname{Res}(\mathcal{P} \unlhd a \unrhd \mathcal{Q})$, and

- if $\mathcal{R} \in \operatorname{Res}(\mathcal{Q})$ and $\mathcal{Q} \in \operatorname{Res}(\mathcal{P})$, then $\mathcal{R} \in \operatorname{Res}(\mathcal{P})$.

Of course, the very idea of a service SHRAT that supports forecasting of the execution of future actions risk in a residual thread $\mathcal{Q} \unlhd$ sh. ok $\unrhd \mathcal{R}$ of $\mathcal{P}$, thus

$$
(\mathcal{Q} \unlhd \operatorname{sh} . \text { ok } \unrhd \mathcal{R}) / \mathrm{sh} \text { SHRAT }
$$

requires that SHRAT is aware of the specification of $\mathcal{Q}$. So, a reply function that only uses the current co-action and those processed before is in this case not sufficient. It seems most natural to model that SHRAT "gets to know and analyzes" $\mathcal{Q}$ 's specification upon the request sh.ok in the useapplication (1) above. We describe this change of state of SHRAT and the resulting reply in the following definition. 
DeFinition 4.1.2. Let a pushdown thread $\mathcal{P}$ be given by some specification $\mathcal{F}_{\mathcal{P}}$ and let sh.ok be the only action in $\mathcal{P}$ with focus sh. Then the service SHRAT is defined by the following two properties:

(1) for any residual thread $\mathcal{Q} \unlhd$ sh.ok $\unrhd \mathcal{R}$ of $\mathcal{P}$,

$$
(\mathcal{Q} \unlhd \mathrm{sh} . \text { ok } \unrhd \mathcal{R}) / \mathrm{sh} \operatorname{SHRAT}=(\mathcal{Q} \unlhd \mathrm{sh} . \mathrm{ok} \unrhd \mathcal{R}) / \mathrm{sh} \operatorname{SHRAT}\left(\mathcal{F}_{\mathcal{P}}, \mathcal{Q}\right),
$$

where $\operatorname{SHRAT}\left(\mathcal{F}_{\mathcal{P}}, \mathcal{Q}\right)$ is the instance of $\operatorname{SHRAT}$ that has loaded $\mathcal{F}_{\mathcal{P}}$ and analyzed $\mathcal{Q}$, and

(2) $(\mathcal{Q} \unlhd$ sh.ok $\unrhd \mathcal{R}) /$ sh $\operatorname{SHRAT}\left(\mathcal{F}_{\mathcal{P}}, \mathcal{Q}\right)=$

$$
\begin{cases}\mathcal{Q} / \mathrm{sh} \text { SHRAT } & \text { (thus reply true) if no risk-action } \\ & \text { will be executed in } \mathcal{Q} / \text { sh SHRAT } \\ \mathcal{R} / \mathrm{sh} \text { SHRAT } & \text { (thus reply false) if a risk-action } \\ & \text { will be executed in } \mathcal{Q} / \text { sh SHRAT. }\end{cases}
$$

The (instantiated) service $\operatorname{SHRAT}\left(\mathcal{F}_{\mathcal{P}}, \mathcal{Q}\right)$ models a "security hazard risk assessment" in the sense that if a security hazard in $\mathcal{Q}$ is modeled by the execution of the action risk, the reply true to $\mathcal{Q} \unlhd$ sh.ok $\unrhd \mathcal{R}$ ensures that in the residual thread $\mathcal{Q} / \mathrm{sh}$ SHRAT no security hazard will occur (cf. [9]).

It can be the case that $\operatorname{SHRAT}\left(\mathcal{F}_{\mathcal{P}}, \mathcal{Q}\right)$ replies true because $\operatorname{SHRAT}$ will reply false to a future sh.ok-test in $\mathcal{Q} /$ sh SHRAT. For example, in the regular thread $P_{1}$ given and depicted below, the various sh.ok-tests are evaluated as follows:

$$
\begin{array}{l|ll}
P_{1}=P_{2} \unlhd \mathrm{sh} . \mathrm{ok} \unrhd P_{8} & (\text { true }) & P_{5}=b \circ P_{2} \\
P_{2}=P_{3} \unlhd a \unrhd P_{4} & & P_{6}=\text { risk } \circ P_{1} \\
P_{3}=P_{5} \unlhd \mathrm{sh} . \mathrm{ok} \unrhd P_{6} & \text { (true) } & P_{7}=c \circ P_{8} \\
P_{4}=P_{6} \unlhd \mathrm{sh} . \mathrm{ok} \unrhd P_{7} & \text { (false) } & P_{8}=\mathrm{S} .
\end{array}
$$
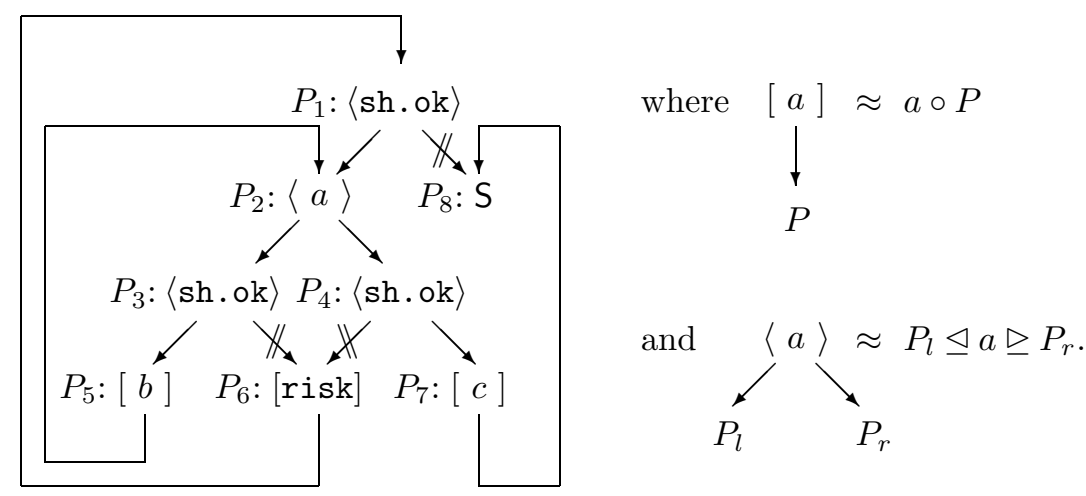

Clearly, the thread $\mathcal{T}=P_{1} /$ sh SHRAT satisfies $\mathcal{T}=b \circ \mathcal{T} \unlhd a \unrhd c \circ \mathrm{S}$. 
In the next section we discuss how to instantiate SHRAT for regular threads in an appropriate way.

4.2. SHRAT for regular threads. Following Convention 2.1.2, we assume that if a regular thread $P_{1}$ is given, it is given by a linear recursive specification $\mathcal{F}_{P_{1}}$ that contains an equation $P_{1}=t_{1}(\vec{P})$. Furthermore, we say that an equation $P_{j}=P_{l} \unlhd a \unrhd P_{r}$ in $\mathcal{F}_{P_{1}}$ has a predecessor if $P_{j}$ occurs in the right-hand side of at least one equation. Finally, we restrict to specifications $\mathcal{F}_{P_{1}}$ with the property that if $P_{j}=P_{l} \unlhd \mathrm{sh}$. ok $\unrhd P_{r} \in \mathcal{F}_{P_{1}}$, then $l \neq r$ (otherwise, the reply to sh.ok would be meaningless).

Starting from $P_{1} /$ sh SHRAT with the regular thread $P_{1}$ specified in $\mathcal{F}_{P_{1}}$, we provide an algorithm that upon each residual thread of the form

$$
\left(P_{m} \unlhd \mathrm{sh} . \text { ok } \unrhd P_{j}\right) / \mathrm{sh} \mathrm{SHRAT}
$$

constructs an instantiated service $\operatorname{SHRAT}\left(\mathcal{F}_{P_{1}}, P_{m}\right)$ that gives the correct reply. Typical for this algorithm is that $\operatorname{SHRAT}\left(\mathcal{F}_{P_{1}}, P_{m}\right)$ contains a copy of $\mathcal{F}_{P_{1}}$ in which all sh.ok actions are annotated with the correct reply. To this end, $\mathcal{F}_{P_{1}}$ is loaded into SHRAT and analyzed as follows: number each equation that contains a risk-occurrence starting from 1 . Then, for each numbered equation label each predecessor equation with the next free number until a connecting sh. ok-equation is found, or a loop occurs, or an equation without predecessors is found. In the case that some sh.ok-equation is found and connects via its true-branch, its sh.ok-action is annotated false $\left(\mathrm{sh} . \mathrm{ok}^{\mathrm{false}}\right)$; if it connects via its false-branch, the equation is labeled with a fresh negative number (it may possibly lead to a risk-action, namely when a false-annotation is added in a future inspection). Then this procedure is repeated for equations labeled with a negative number, again instantiating first occurrences of sh.ok-actions with false if their true-branch leads to an action risk. Finally, all non-annotated sh.okactions are annotated true because their true-branch does not lead to a risk-action.

In Figure 1, we illustrate how the annotation proceeds: first the two lowest sh.ok actions are annotated false, and because of the $\searrow$ arrow, the equation of the leftmost one is labeled with a fresh negative number. The combination of the false-annotation and this label leads to the falseannotation of the topmost sh.ok-action.

Construction of $\operatorname{SHRAT}\left(\mathcal{F}_{P_{1}}, P_{m}\right)$ for a regular thread $P_{1}$. Let $\mathcal{F}_{P_{1}}=$ $\left\{P_{i}=t_{i}(\vec{P}) \mid i=1, \ldots, n\right\}$ be a linear specification of the regular thread $P_{1}$. Upon a residual thread

$$
P_{m} \unlhd \text { sh.ok } \unrhd P_{w}
$$

the service $\operatorname{SHRAT}\left(\mathcal{F}_{P_{1}}, P_{m}\right)$ is constructed as follows: load $\mathcal{F}_{P_{1}}$ in SHRAT. We further call this copy $\mathcal{F}_{P_{1}}^{\text {an }}$. Label each equation in $\mathcal{F}_{P_{1}}^{\text {an }}$ that contains 


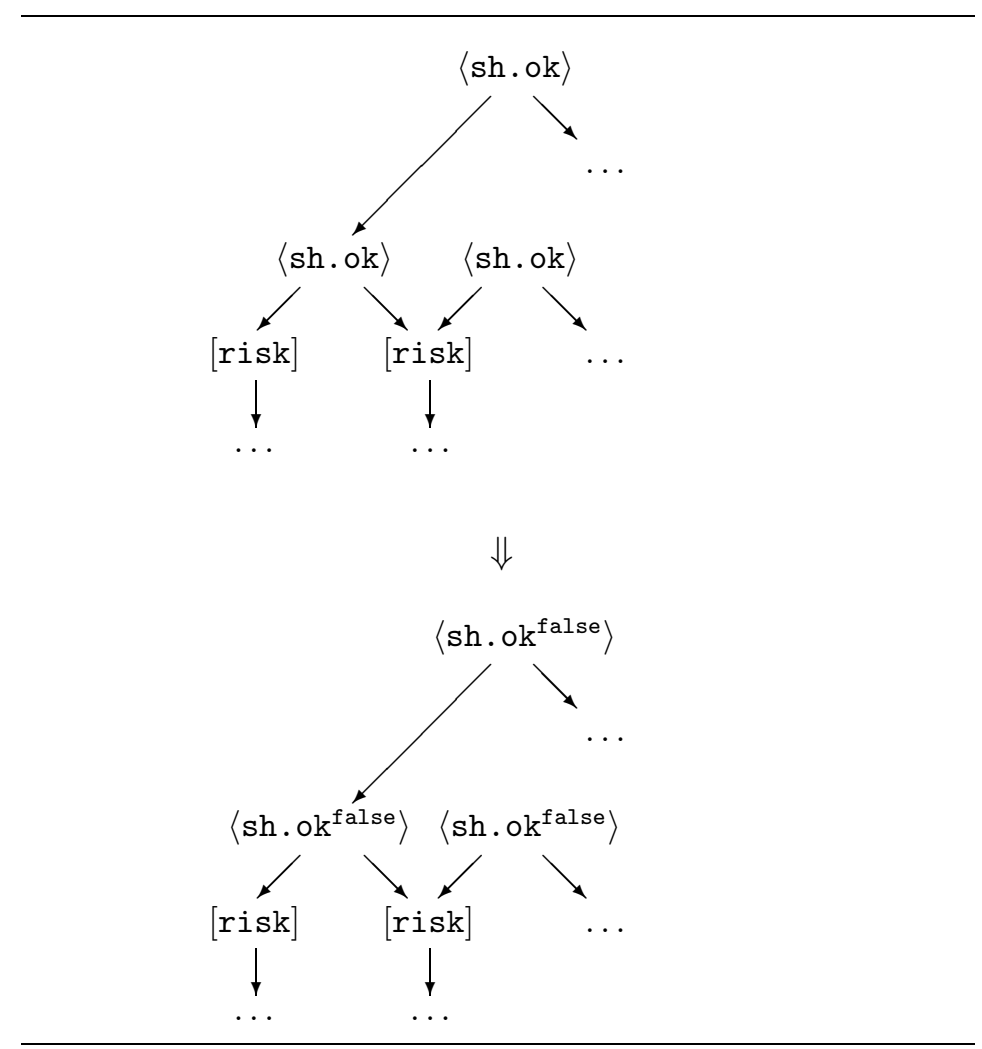

Figure 1. Annotating sh.ok actions

risk in the right-hand side with a number, starting from 1 , say $1, \ldots, k$. If no risk-actions occur in $\mathcal{F}_{P_{1}}^{\text {an }}$, then apply step 3 below. In the other case, apply step 1:

1. On $\mathcal{F}_{P_{1}}^{\text {an }}$ apply the procedure $\operatorname{Eval}^{+}(1)$, where $\operatorname{Eval}^{+}(i)$ for $i \geq 1$ is defined as follows:

Eval $^{+}(i)$ : If the equation labeled with number $i$ has the form

$$
\text { (i) } P_{j}=P_{l} \unlhd a \unrhd P_{r}
$$

then evaluate all $P_{j}$ occurrences in the right-hand sides of all equations, i.e., apply steps (1a) - (1e) below exhaustively, where evaluation goes with some bookkeeping: we will in some cases give equations a next free number and possibly annotate sh.ok-actions with false. The first free positive number is $k+1$ and the first free negative number is -1 . Furthermore, the next free number for positive numbers is 
the smallest $p>0$ not already used, and for negative numbers the largest $p<0$ not already used:

(a) No non-evaluated $P_{j}$ occurrences left: if there is an equation numbered $i+1$ then apply $\mathrm{Eval}^{+}(i+1)$, else, if negative numbers are used, go to step 2; if none of these is the case, go to step 3,

(b) If $P_{v}=P_{j} \unlhd \mathrm{sh} . \mathrm{ok} \unrhd P_{q}$, then replace sh.ok by sh.ok ${ }^{\text {false }}$ and search the next non-evaluated $P_{j}$ occurrence (a possible number of this equation is preserved),

(c) If $P_{v}=P_{q} \unlhd \mathrm{sh} . \mathrm{ok} \unrhd P_{j}$ and this equation is not numbered, then give it the next free negative number and search the next nonevaluated $P_{j}$ occurrence, else just search the next non-evaluated $P_{j}$ occurrence,

(d) If $P_{v}=P_{q} \unlhd \mathrm{sh} . \mathrm{ok}^{\mathrm{false}} \unrhd P_{j}$ and this equation is not numbered, then give it the next free negative number and search the next non-evaluated $P_{j}$ occurrence, else just search the next nonevaluated $P_{j}$ occurrence,

(e) All remaining cases, i.e., equations of the form $P_{v}=P_{j} \unlhd b \unrhd P_{q}$ or $P_{v}=P_{q} \unlhd b \unrhd P_{j}$ : if not yet numbered, give this equation the next free positive number and search the next non-evaluated $P_{j}$ occurrence; else, just search the next non-evaluated $P_{j}$ occurrence.

2. On $\mathcal{F}_{P_{1}}^{\text {an }}$ apply the procedure $\operatorname{Eval}^{-}(-1)$, where $\operatorname{Eval}^{-}(i)$ for $i \leq-1$ is defined as follows:

$\operatorname{Eval}^{-}(i)$ :

- if the equation labeled with number $i$ has the form

$$
\text { (i) } P_{j}=P_{l} \unlhd \mathrm{sh} . \mathrm{ok} \unrhd P_{r},
$$

then apply $\mathrm{Eval}^{-}(i-1)$ if there is an equation numbered $i-1$, otherwise go to step 3 ;

- if the equation labeled with number $i$ has the form

$$
\text { (i) } P_{j}=P_{l} \unlhd a \unrhd P_{r} \text { for } a \neq \mathrm{sh} . \mathrm{ok}
$$

(possibly $a=\mathrm{sh} . \mathrm{ok}^{\mathrm{false}}$ ), then evaluate all $P_{j}$ occurrences in the right-hand sides of all equations, i.e., apply steps (2a) - (2e) below exhaustively, where evaluation again goes with some bookkeeping: we will in some cases give equations the next free negative number and possibly annotate sh.ok-actions with false:

(a) No non-evaluated $P_{j}$ occurrences left: if there is an equation numbered $i-1$ then apply $\operatorname{Eval}^{-}(i-1)$, else go to step 3,

(b) If $P_{v}=P_{j} \unlhd$ sh.ok $\unrhd P_{q}$, then replace sh.ok by sh.ok ${ }^{\text {false }}$ and search the next non-evaluated $P_{j}$ occurrence (a possible number of this equation is preserved),

(c) If $P_{v}=P_{q} \unlhd \mathrm{sh}$.ok $\unrhd P_{j}$, then search the next non-evaluated $P_{j}$ occurrence, 
(d) If $P_{v}=P_{q} \unlhd \mathrm{sh} . \mathrm{ok}^{\mathrm{false}} \unrhd P_{j}$ and this equation is not numbered, then give it the next free negative number and search the next non-evaluated $P_{j}$ occurrence, else just search the next nonevaluated $P_{j}$ occurrence,

(e) All remaining cases, i.e., equations of the form $P_{v}=P_{j} \unlhd b \unrhd P_{q}$ or $P_{v}=P_{q} \unlhd b \unrhd P_{j}$ : if not yet numbered, give this equation the next free negative number and search the next non-evaluated $P_{j}$ occurrence; else, just search the next non-evaluated $P_{j}$ occurrence.

3. Replace all sh.ok occurrences in $\mathcal{F}_{P_{1}}^{\text {an }}$ that are not yet annotated by sh.ok ${ }^{\text {true }}$.

Now $\operatorname{SHRAT}\left(\mathcal{F}_{P_{1}}, P_{m}\right)$ is defined as the service that replies to the residual thread $P_{m} \unlhd \mathrm{sh} . \mathrm{ok} \unrhd P_{w}$ with the annotation $b$ found in the right-hand side $P_{m} \unlhd$ sh. ok ${ }^{b} \unrhd P_{w}$ of its internal specification $\mathcal{F}_{P_{1}}^{\mathrm{an}}$.

TheOREM 4.2.1. Let $P_{1}$ be a regular thread specified by the linear recursive specification $\mathcal{F}_{P_{1}}$. Then, upon each residual thread of the form

$$
P_{m} \unlhd \text { sh.ok } \unrhd P_{w},
$$

the tool $\operatorname{SHRAT}\left(\mathcal{F}_{P_{1}}, P_{m}\right)$ is sound, i.e., agrees with Definition 4.1.2.

Hence,

$$
\begin{aligned}
\left(P_{m}\right. & \left.\unlhd \mathrm{sh} . \mathrm{ok} \unrhd P_{w}\right) / \mathrm{sh} \text { SHRAT } \\
& =\left(P_{m} \unlhd \mathrm{sh} . \mathrm{ok} \unrhd P_{w}\right) / \mathrm{sh} \operatorname{SHRAT}\left(\mathcal{F}_{P_{1}}, P_{m}\right) \\
& =\left\{\begin{array}{l}
P_{m} / \mathrm{sh} \mathrm{SHRAT} \quad \text { if } P_{m} / \mathrm{sh} \operatorname{SHRAT} \text { does not execute } \text { risk, } \\
P_{w} / \mathrm{sh} \mathrm{SHRAT} \quad \text { otherwise. }
\end{array}\right.
\end{aligned}
$$

Proof. Assume $P_{m} \unlhd$ sh.ok $\unrhd P_{w}$ is a residual thread of $P_{1}$. Clearly the algorithm for $\operatorname{SHRAT}\left(\mathcal{F}_{P_{1}}, P_{m}\right)$ terminates and $P_{m} \unlhd \mathrm{sh} . \mathrm{ok}^{b} \unrhd P_{w}$ occurs at least once as a right-hand side in $\mathcal{F}_{P_{1}}^{\text {an }}$ (in case of multiple occurrences, $b$ has the same value). We argue that the boolean $b$ is the correct reply to

$$
\left(P_{m} \unlhd \text { sh.ok } \unrhd P_{w}\right) / \text { sh } \operatorname{SHRAT}\left(\mathcal{F}_{P_{1}}, P_{m}\right) .
$$

In case $\mathcal{F}_{P_{1}}^{\text {an }}$ contains no risk action, all annotations are true (step 3 ), which obviously is correct.

In case $\mathcal{F}_{P_{1}}^{\text {an }}$ contains at least one risk action, it is clear that after all $\mathrm{Eval}^{+}(i)$ 's have been applied (step 1), all true-branches of annotated sh. $\mathrm{ok}^{\mathrm{false}}$ actions lead to risk. Furthermore, the right-hand sides of all negatively numbered equations have a sh.ok action (possibly annotated false) of which the false-branch leads to risk. At Eval ${ }^{-}(i)$ (step 2), the negatively numbered equations with non-annotated action sh.ok will not be annotated false (as their true-branch does not lead to risk). The remaining labeled equations all have a residual thread that may lead to risk, and thus yield next (negative) numbers until a loop occurs, or an equation 
without a predecessor is found, or another sh. ok that connects via its truebranch occurs (in the latter case, this action is annotated false). Hence, after step 3, all annotations are correct.

4.3. SHRAT for pushdown threads. It is not clear how to define a (terminating) algorithm for SHRAT that is correct for arbitrary pushdown threads. However, in the particular case that either no test action sh.ok or no action risk is executed by a pushdown thread $\mathcal{P}$, the correct reply of sh.ok in

$$
(\mathcal{P} \unlhd \text { sh.ok } \unrhd \mathcal{Q}) / \mathrm{sh} \text { SHRAT }
$$

follows easily from Theorem 3.2.1 (i.e., equivalence of pushdown threads is decidable): consider a pushdown thread

$$
P_{k} / \mathrm{s} S(\alpha)
$$

where $P_{k}$ is specified in $\mathcal{F}$. Assuming that the action $a^{\prime}$ does not occur in $\mathcal{F}$, define $\mathcal{F}^{a^{\prime}}$ by replacing in $\mathcal{F}$ each occurrence of the action $a$ by $a^{\prime}$ and replacing all identifiers $P_{i}$ by $P_{i}^{a^{\prime}}$. Then $P_{k} / \mathrm{s} S(\alpha)$ does not execute $a$ if and only if $P_{k} / \mathrm{s} S(\alpha)=P_{k}^{a^{\prime}} / \mathrm{s} S(\alpha)$, so this is decidable. Note that if $P_{k} / \mathrm{s} S(\alpha)=P_{k}^{a^{\prime}} / \mathrm{s} S(\alpha)$, then for any residual thread $P_{l} / \mathrm{s} S(\beta)$ of $P_{k} / \mathrm{s} S(\alpha)$, also $P_{l} / \mathrm{s} S(\beta)=P_{l}^{a^{\prime}} / \mathrm{s} S(\beta)$.

A pushdown thread $\mathcal{P}=P_{k} / \mathrm{s} S(\alpha)$ is called shrat-safe if either $\mathcal{P}=$ $P_{k}^{\text {risk }^{\prime}} / \mathrm{s} S(\alpha)$ or $\mathcal{P}=P_{k}^{\text {sh. ok }} / \mathrm{s} S(\alpha)$. In both cases the correct reply to sh.ok in

$$
\mathcal{P} \unlhd \operatorname{sh} . \text { ok } \unrhd \mathcal{Q}
$$

can be found:

- if $\mathcal{P}=P_{k}^{\text {risk' }} / \mathbf{s} S(\alpha)$, then this reply is true, thus

$$
(\mathcal{P} \unlhd \text { sh.ok } \unrhd \mathcal{Q}) / \mathrm{sh} \text { SHRAT }=\mathcal{P} / \text { sh SHRAT, }
$$

- if $\mathcal{P}=P_{k}^{\text {sh. ok }} / \mathrm{s} S(\alpha)$, then both replies can occur, thus

$$
\begin{aligned}
(\mathcal{P} & \unlhd \text { sh.ok } \unrhd \mathcal{Q}) / \mathrm{sh} \text { SHRAT } \\
& = \begin{cases}\mathcal{P} / \mathrm{sh} \text { SHRAT } & \text { (reply true) if } P_{k} / \mathrm{s} S(\alpha)=P_{k}^{\mathrm{risk}^{\prime}} / \mathrm{s} S(\alpha), \\
\mathcal{Q} / \mathrm{sh} \text { SHRAT } & \text { otherwise, }\end{cases}
\end{aligned}
$$

where the latter case is only meaningful if $\mathcal{Q}$ is also shrat-safe.

Although much weaker, it is not unreasonable to consider shrat-safe pushdown threads. This situation can always be obtained: upon a residual thread $(\mathcal{P} \unlhd$ sh. ok $\unrhd \mathcal{Q}) /$ sh SHRAT, rename all sh. ok actions in the specification of $\mathcal{P}$, thus ignoring their forecasting effect and evaluating both their true and false-branches. If SHRAT then replies true, this certainly 
comprises a security hazard risk assessment of $\mathcal{P}$. The only problem is that if SHRAT replies false, it is not certain that $\mathcal{P}$ will indeed execute risk.

§5. Digression and discussion. In this paper we presented some of our latest work on thread algebra and on security hazard risk assessment (as defined in [9]). We end the paper with a few comments on the latter subject.

5.1. Architecture-sensitive services. First, we propose to call services as SHRAT architecture-sensitive services: in case SHRAT has to reply to a thread

$$
\mathcal{Q} \unlhd \operatorname{sh} . \mathrm{ok} \unrhd \mathcal{R},
$$

it first needs to analyze the future behaviour of $\mathcal{Q}$ and therefore it needs to "know" both the specification and the particular execution state. Assuming that $\mathcal{Q}$ is specified in $\mathcal{F}_{\mathcal{P}}$, this idea is captured in Definition 4.1 .2 by the equation

$$
(\mathcal{Q} \unlhd \operatorname{sh} . \text { ok } \unrhd \mathcal{R}) / \mathrm{sh} \operatorname{SHRAT}=(\mathcal{Q} \unlhd \operatorname{sh} . \text { ok } \unrhd \mathcal{R}) / \mathrm{sh} \operatorname{SHRAT}\left(\mathcal{F}_{\mathcal{P}}, \mathcal{Q}\right),
$$

which characterizes the instantiation of $\operatorname{SHRAT}$ to $\operatorname{SHRAT}\left(\mathcal{F}_{\mathcal{P}}, \mathcal{Q}\right)$.

So, in the particular case of SHRAT (and similar services such as rational agents discussed in [8]), the reply in a use-application is architecturesensitive and can not be defined with a reply function that only depends on the current co-action and those processed before (such as the reply function for the stack defined in Example 2.2.1). Typically, different use-applications need not commute if architecture-sensitive services are involved, e.g.,

$$
([(\text { risk } \circ \mathrm{S} \unlhd \mathrm{s} . \mathrm{pop} \unrhd \mathrm{S}) \unlhd \mathrm{sh} . \mathrm{ok} \unrhd \mathrm{D}] / \mathrm{sh} \mathrm{SHRAT}) / \mathrm{s} S(\epsilon)=\mathrm{D}
$$

while

$$
([(\text { risk } \circ \mathrm{S} \unlhd \mathrm{s} . \text { pop } \unrhd \mathrm{S}) \unlhd \mathrm{sh} . \mathrm{ok} \unrhd \mathrm{D}] / \mathrm{s} S(\epsilon)) / \mathrm{sh} \mathrm{SHRAT}=\mathrm{S} .
$$

Use-applications with services with a reply function that only depends on the current co-action and those processed before do commute if distinct foci are used (cf. [7]).

5.2. SHRAT for pushdown threads. At this stage, it is not clear how to define a (terminating) algorithm for SHRAT that is correct for all pushdown threads. One possibility may be to approximate pushdown threads by regular threads in such a way that a sound risk-analysis can be established. Given a linear specification $\mathcal{F}_{P_{1}}$ of $P_{1}$ and a stack $S$, it seems likely that in $P_{1} / \mathrm{s} S(\alpha)$ only finitely many stack configurations (uniformly depending on $\mathcal{F}_{P_{1}}$ and $\alpha$ ) play a distinctive role with respect to SHRAT's replies. 
Another approach is to start from a game theoretic characterization of SHRAT: in residual threads of the form

$$
(\mathcal{Q} \unlhd \text { sh.ok } \unrhd \mathcal{R}) / \text { sh SHRAT, }
$$

the service SHRAT has to give the correct reply (according to its Definition 4.1.2), while the opponent replies to all other test actions and aims for the execution of risk. We do not (yet) know whether game theoretic results cover this particular game. Hence:

Open question: Is SHRAT decidable for all pushdown threads?

An interesting simplification may be the case of one-counter threads, i.e., regular threads that use a counter (a stack over a singleton datatype) instead of a stack, with s.push and s.pop as the only actions. Also for this case, the above question is still open.

Of course, security hazard risk assessment for computable threads is undecidable. In the setting of Turing machines, given a regular control program $P$ and tape configuration Tape $(\alpha \hat{x} \beta)$ with head pointing at $x$, it is undecidable whether some action of $P$ will be executed in $P / \operatorname{tmt} \operatorname{Tape}(\alpha \hat{x} \beta)$ : there is a straightforward reduction to the halting problem (cf. [8]).

5.3. SHRAT and external services. In order to define security hazard risk assessment in precisely the same way as was done in [9], the results and explanations for both the regular and the pushdown case in Section 4 should be slightly modified. In [9], a thread can also engage in external communication with a service $E$ (via actions with focus $e$ ). Such a communication blocks further assessment of SHRAT because $E$ is beyond control of the thread under execution. It is not difficult to implement this modification in the algorithm for regular threads: in the evaluation step, simply stop evaluation upon an equation defined by a postconditional composition over e.m. However, for clarity of presentation we did not consider this possibility before.

\section{REFERENCES}

[1] J.W. DE BAKKer and J.I. Zucker, Processes and the denotational semantics of concurrency, Information and Control, vol. 54 (1982), no. 1/2, pp. 70-120.

[2] J.A. Bergstra and I. Bethke, Polarized process algebra and program equivalence, Automata, languages and programming, Proceedings 30th ICALP, Eindhoven, The Netherlands (J.C.M. Baeten, J.K. Lenstra, J. Parrow, and G.J. Woeginger, editors), LNCS, vol. 2719, Springer-Verlag, 2003, pp. 1-21.

[3] J.A. Bergstra, I. Bethke, and A. Ponse, Decision problems for pushdown threads, Electronic report PRG0502, Faculty of Science, University of Amsterdam, 2005, available at www.science.uva.nl/research/prog/publications.html.

[4] J.A. Bergstra and J.W. Klop, Process algebra for synchronous communication, Information and Control, vol. 60 (1984), no. 1/3, pp. 109-137. 
[5] J.A. Bergstra and M.E. Loots, Program algebra for sequential code, Journal of Logic and Algebraic Programming, vol. 51 (2002), no. 2, pp. 125-156.

[6] J.A. Bergstra and C.A. Middelburg, A thread algebra with multi-level strategic interleaving, Proceedings CiE 2005 (S.B. Cooper, B. Loewe, and L. Torenvliet, editors), LNCS, vol. 3526, Springer-Verlag, 2005, pp. 35-48.

[7] J.A. Bergstra and A. Ponse, Combining programs and state machines, Journal of Logic and Algebraic Programming, vol. 51 (2002), no. 2, pp. 175-192.

[8] —, Execution architectures for program algebra, Technical report Logic Group Preprint Series 230, Department of Philosophy, Utrecht University, 2004, to appear in the Journal of Applied Logic, prior version available at http://www.phil.uu. $\mathrm{nl} /$ preprints/lgps/?lang=en.

[9] - A bypass of Cohen's impossibility result, Advances in grid computing - EGC 2005 (P.M.A. Sloot, A.G. Hoekstra, T. Priol, A. Reinefeld, and M. Bubak, editors), LNCS, vol. 3470, Springer-Verlag, 2005, also vailable as Electronic report PRG0501 at www.science.uva.nl/research/prog/publications.html, pp. 1097-1106.

[10] O. Burkart and B. Steffen, Pushdown processes: Parallel composition and model checking, Concur'94, LNCS, vol. 836, Springer-Verlag, August 1994, pp. 98-113.

[11] F. Cohen, Computer viruses - theory and experiments, Computers 8 Security, vol. 6 (1984), no. 1, pp. 22-35, also available at http://vx.netlux.org/lib/afc01.html.

[12] S.A. Greibach, Theory of program structures: Schemes, semantics, verification, LNCS, vol. 36, Springer-Verlag, 1975.

[13] P. JanČAR, F. Moller, and Z. SAWA, Simulation problems for one-counter machines, Proceedings of SOFSEM'99: The 26th Seminar on Current Trends in Theory and Practice of Informatics, LNCS, vol. 1725, Springer-Verlag, 1999, pp. 398407.

[14] Z. Manna, Mathematical theory of computation, McGraw-Hill, New-York, 1974.

[15] G. SÉnizergues, $L(A)=L(B)$ ?, Technical report 1161-97, LaBRI, Université Bordeaux, 1997, available at www.labri.u-bordeaux.fr.

$[16]-, L(A)=L(B)$ ? decidability results from complete formal systems, Theoretical Computer Science, vol. 251 (2001), pp. 1-166.

[17] C. StiRling, Decidability of bisimulation equivalence for pushdown processes, Technical report EDI-INF-RR0005, Laboratory for Foundations of Computer Science, University of Edinburgh, 2000, available at http://www.inf.ed.ac.uk/research/lfcs/ publications.html.

[18] - Decidability of dpda equivalence, Theoretical Computer Science, vol. 255 (2001), pp. 21-31. 
PROGRAMMING RESEARCH GROUP, FACULTY OF SCIENCE UNIVERSITY OF AMSTERDAM, THE NETHERLANDS and

APPLIED LOGIC GROUP, DEPARTMENT OF PHILOSOPHY UTRECHT UNIVERSITY, THE NETHERLANDS

$U R L:$ www.science.uva.nl/ janb/

PROGRAMMING RESEARCH GROUP, FACULTY OF SCIENCE UNIVERSITY OF AMSTERDAM, THE NETHERLANDS $U R L$ : www.science.uva.nl/ inge/

PROGRAMMING RESEARCH GROUP, FACULTY OF SCIENCE UNIVERSITY OF AMSTERDAM, THE NETHERLANDS $U R L$ : www.science.uva.nl/ alban/ 